\title{
Altered gene expression in conjunctival squamous cell carcinoma
}

\author{
Alka Mahale ${ }^{1}$, Hind Alkatan ${ }^{2}$, Saeed Alwadani ${ }^{1,2,3}$, Maha Othman ${ }^{1}$, Maria J Suarez ${ }^{3}$, \\ Antoinette Price ${ }^{4}$, Hailah Al-Hussain ${ }^{1}$, Sabah Jastaneiah ${ }^{1}$, Wayne Yu $^{5}$, Azza Maktabi ${ }^{1}$, \\ Edward P Deepak ${ }^{1,3}$, Charles G Eberhart ${ }^{3,4,6}$ and Laura Asnaghi ${ }^{4}$
}

${ }^{1}$ King Khaled Eye Specialist Hospital, Riyadh, Saudi Arabia; ${ }^{2}$ Department of Ophthalmology, King Saud University, Riyadh, Saudi Arabia; ${ }^{3}$ Department of Ophthalmology, Johns Hopkins University, School of Medicine, Baltimore, MD, USA; ${ }^{4}$ Department of Pathology, Johns Hopkins University, School of Medicine, Baltimore, MD, USA; ${ }^{5}$ Microarray Core Facility, Sidney Kimmel Cancer Center, Johns Hopkins University, School of Medicine, Baltimore, MD, USA and ${ }^{6}$ Department of Oncology, Johns Hopkins University, School of Medicine, Baltimore, MD, USA

\begin{abstract}
Conjunctival squamous cell carcinoma is a malignancy of the ocular surface. The molecular drivers responsible for the development and progression of this disease are not well understood. We therefore compared the transcriptional profiles of eight snap-frozen conjunctival squamous cell carcinomas and one in situ lesion with normal conjunctival specimens in order to identify diagnostic markers or therapeutic targets. RNA was analyzed using oligonucleotide microarrays, and a wide range of transcripts with altered expression identified, including many dysregulated in carcinomas arising at other sites. Among the upregulated genes, we observed more than 30-fold induction of the matrix metalloproteinases, MMP-9 and MMP-11, as well as a prominent increase in the mRNA level of a calcium-binding protein important for the intracellular calcium signaling, S100A2, which was induced over 20 -fold in the tumor cohort. Clusterin was the most downregulated gene, with an approximately 180-fold reduction in the mRNA expression. These alterations were all confirmed by qPCR in the samples used for initial microarray analysis. In addition, immunohistochemical analysis confirmed the overexpression of MMP-11 and S100A2, as well as reductions in clusterin, in several independent in situ carcinomas of conjunctiva. These data identify a number of alterations, including upregulation of MMP-9, MMP-11, and S100A2, as well as downregulation of clusterin, associated with epithelial tumorigenesis in the ocular surface.
\end{abstract}

Modern Pathology (2016) 29, 452-460; doi:10.1038/modpathol.2016.41; published online 26 February 2016

Conjunctival squamous cell carcinoma generally arises from the bulbar conjunctiva and limbus. It is the most advanced type of ocular surface squamous neoplasia, which also includes conjunctival intraepithelial neoplasia, and conjunctival carcinoma in situ. ${ }^{1}$ Squamous carcinomas are the commonest non-pigmented malignancies of the conjunctiva, with a yearly incidence that ranges from 0.02 to 3.5 per $100000 .^{2}$ One of the main risk factors is exposure to the high ultraviolet (UV-B) light, thus the incidence of conjunctival squamous cell carcinoma has a geographic variability and is highest at shorter

Correspondence: Professor CG Eberhart or Dr L Asnaghi, Department of Pathology, Johns Hopkins University, School of Medicine, Smith Building, 400 N. Broadway Avenue, Baltimore, MD 21287, USA.

E-mail: ceberha@jhmi.edu or lasnagh1@jhmi.edu

Received 21 September 2015; revised 13 January 2016; accepted 14 January 2016; published online 26 February 2016 distances from the equator. Another important risk factor is immunosuppression, often due to human immunodeficiency virus (HIV) infection. ${ }^{3,4}$ The role of human papilloma virus (HPV) in the pathogenesis of conjunctival squamous cell carcinoma remains controversial. 5,6

The tumors are locally invasive, with intraocular and orbital involvement reported in $2-15 \%$ and $12-16 \%$ of cases, respectively. ${ }^{7,8}$ It rarely spreads to distant organs, with occasional metastases to regional lymph nodes. Primary therapy is surgical removal. Cryotherapy, radiation, and chemotherapy have been used after excision to reduce recurrence rates. Topical mitomycin C, ${ }^{9}$ 5-fluorouracil, ${ }^{10}$ and interferon alpha-2 $b^{11,12}$ have been successfully used for recurrent lesions.

From a biological standpoint, little is known about the molecular drivers responsible for the formation and progression of conjunctival squamous cell carcinoma. Inherited mutations in DNA repair 
Table 1 Clinical and pathological characteristics of the ocular surface squamous neoplasia specimens

\begin{tabular}{|c|c|c|c|c|c|c|}
\hline Case & Sex & Vascularized & Histopathology & $\begin{array}{l}\text { Invasion outside } \\
\text { conjunctiva }\end{array}$ & $\begin{array}{l}\text { Subsequent } \\
\text { recurrence }\end{array}$ & Immunosuppression \\
\hline 1 & $\mathrm{M}$ & Yes & Conjunctival carcinoma in situ & Cornea & No & Unknown \\
\hline 2 & $\mathrm{M}$ & Yes & Conjunctival squamous cell carcinoma & Cornea orbit & No & No \\
\hline 3 & M & Yes & Conjunctival squamous cell carcinoma & Cornea & No & No \\
\hline 5 & $\mathrm{~F}$ & Yes & Conjunctival squamous cell carcinoma & Cornea orbit & No & No \\
\hline 7 & $\mathrm{M}$ & Unknown & Conjunctival squamous cell carcinoma & Unknown & No & Unknown \\
\hline 10 & M & Unknown & $\begin{array}{l}\text { Conjunctival squamous cell carcinoma } \\
\text { with focal adnexal differentiation }\end{array}$ & Orbit & No & No \\
\hline 12 & $\mathrm{M}$ & Yes & Conjunctival squamous cell carcinoma & Cornea & Yes & No \\
\hline 13 & $\mathrm{M}$ & Yes & $\begin{array}{l}\text { Poorly differentiated recurrent } \\
\text { conjunctival squamous cell carcinoma }\end{array}$ & Cornea sclera orbit & No & Unknown \\
\hline 14 & M & Yes & $\begin{array}{l}\text { Recurrent conjunctival squamous cell } \\
\text { carcinoma }\end{array}$ & Sclera orbit & Yes & No \\
\hline
\end{tabular}

Abbreviations: F, Female; M, male. The numerical progression of the case number corresponds to the cases analyzed in our previous report. ${ }^{15}$

enzymes can increase the incidence of these tumors, and p53 alterations have also been reported. ${ }^{13,14}$ We previously documented DNA copy number alterations in a cohort of 10 snap-frozen conjunctival squamous cell carcinoma tumor specimens and two in situ carcinomas by array-based comparative genomic hybridization assay, which allowed us to determine the most frequent DNA gains and losses. ${ }^{15}$ We now compare transcriptional profiles of nine of these tumor specimens from a single center in Saudi Arabia to normal conjunctiva in order to identify dysregulated mRNAs representing molecular targets potentially useful for diagnosis and/or therapy.

\section{Materials and methods}

\section{Clinical Specimens}

Eight conjunctival squamous cell carcinomas and one conjunctival carcinoma in situ cases were identified through review of pathology and tumor bank records at King Khaled Eye Specialist Hospital, Riyadh, Saudi Arabia. Only tumors with tissue snap-frozen at the time of surgery were used in this study. Three ophthalmic pathologists (Drs Alkatan, Edward, and Maktabi) reviewed the histopathological slides in all cases to confirm the presence of carcinoma. With Institutional Review Board approval, relevant clinical data were abstracted from the clinical record and linked to the frozen research specimens using anonymized sample identification numbers. The clinical characteristics of these cases are summarized in Table 1. Two normal conjunctiva and one limbal specimen resected from autopsy eyes at the Johns Hopkins Hospital were used as controls for gene expression profiling. We also examined formalin-fixed paraffin-embedded surgical specimens resected at Johns Hopkins Wilmer Eye Institute with a diagnosis of conjunctival intraepithelial neoplasia with severe dysplasia or conjunctival squamous cell carcinoma using immunohistochemistry.

\section{RNA Extraction}

Immediately after resection, the specimens were transported to the laboratory on ice, snap-frozen in liquid nitrogen, and stored at $-80^{\circ} \mathrm{C}$ until RNA extraction. Total RNA was isolated from frozen tumor tissues using an RNeasy Mini Kit (Qiagen, Germantown, $\mathrm{MD}$, USA) according to the manufacturer's protocol. DNase enzyme digestion was performed as per the manufacturer's instructions to exclude genomic DNA contamination. RNA concentration and quality were determined using a NanoDrop 1000 spectrophotometer (Thermo Scientific, Waltham, MA, USA).

\section{Microarray Analysis}

Microarray assay was performed using SurePrint Agilent human GE $4 \times 44 \mathrm{~K}$ version 2 one-color expression array (Agilent Technologies, Santa Clara, CA, USA). RNA quality was assessed by bioanalyzer (2100 Bioanalyzer, Agilent) and $400 \mathrm{ng}$ of RNA was used for cDNA synthesis. Array hybridizations were carried out at the Microarray Core Facility of the Sidney Kimmel Cancer Center in Johns Hopkins University. Microarray raw data were analyzed using GeneSpring 12.6 software (Agilent). $P$-values in Tables 2 and 3 were calculated using Moderated t-test. $P$-values lower than 0.05 were considered statistically significant. Ingenuity Pathway Analysis (IPA) software (Qiagen) was used to determine the main signaling pathways that are regulated by the genes overexpressed or downregulated in the gene expression profile analysis performed in the conjunctival squamous cell carcinoma samples, compared with normal conjunctiva. $P$-values in Table 4 were calculated using Fisher's Exact test.

\section{Quantitative Real-Time PCR}

The mRNA levels of MMP-9, MMP-11, and S100A2 were also analyzed by quantitative real-time PCR (qPCR). Two hundred nanograms of RNA were used 
Table 2 Genes upregulated in ocular surface squamous neoplasia compared with normal conjunctiva; fold change (FC) $>10$

\begin{tabular}{|c|c|c|c|c|}
\hline P-value & $F C(a b s)$ & Gene symbol & Chromosome & Gene name \\
\hline 0.00490036 & 48.10617 & $\mathrm{CD} 24$ & chrY & CD24 molecule \\
\hline 0.00120449 & 37.94295 & MMP-9 & $\operatorname{chr} 20$ & Matrix metallopeptidase 9 \\
\hline $2.48 \mathrm{E}-05$ & 34.33098 & TMEM132A & chr11 & Transmembrane protein 132A \\
\hline 0.00193533 & 33.137703 & COL1A1 & $\operatorname{chr} 17$ & Collagen, type I, alpha 1 \\
\hline 2.32E-04 & 32.71715 & MMP-11 & $\operatorname{chr} 22$ & Matrix metallopeptidase 11 (stromelysin 3) \\
\hline $6.60 \mathrm{E}-06$ & 25.115358 & CENPF & chr1 & Centromere protein F, 350/400kDa (mitosin) \\
\hline $1.16 \mathrm{E}-05$ & 25.001253 & SCD & chr10 & Stearoyl-CoA desaturase (delta-9-desaturase) \\
\hline 2.66E-04 & 22.138256 & S100A2 & $\operatorname{chr} 1$ & S100 calcium-binding protein A2 \\
\hline 0.00146693 & 21.938208 & HK2 & chr2 & Hexokinase 2 \\
\hline 0.00267661 & 18.583275 & KRT17 & $\operatorname{chr} 17$ & Keratin 17 \\
\hline 2.85E-06 & 18.415516 & CKS2 & chr9 & CDC28 protein kinase regulatory subunit 2 \\
\hline 2.43E-05 & 18.185926 & UBE2C & $\operatorname{chr} 20$ & Ubiquitin-conjugating enzyme E2C \\
\hline 0.00293024 & 17.505316 & CYTH4 & $\operatorname{chr} 22$ & Cytohesin 4 \\
\hline 0.00261672 & 15.982199 & LGALS7 & chr19 & Lectin, galactoside-binding, soluble, 7 \\
\hline $3.48 \mathrm{E}-06$ & 15.73342 & RECQL4 & chr8 & RecQ protein-like 4 \\
\hline 5.47E-04 & 13.731016 & AK4 & chr1 & Adenylate kinase 4 \\
\hline 0.0016305 & 13.466698 & KRT42P & $\operatorname{chr} 17$ & Keratin 42 pseudogene \\
\hline 0.00433193 & 12.890687 & STAT1 & chr2 & Signal transducer and activator of transcription 1 \\
\hline 0.00145267 & 11.87778 & TK1 & $\operatorname{chr} 17$ & Thymidine kinase 1 , soluble \\
\hline $6.66 \mathrm{E}-05$ & 11.28387 & CDCA8 & chr1 & Cell division cycle associated 8 \\
\hline $2.05 \mathrm{E}-04$ & 11.265495 & C17orf96 & $\operatorname{chr17}$ & Chromosome 17 open reading frame 96 \\
\hline 0.00195755 & 11.210659 & TNFRSF12A & $\operatorname{chr} 16$ & Tumor necrosis factor receptor superfamily, 12A \\
\hline 0.00447209 & 11.119723 & $\mathrm{CDH} 3$ & chr16 & Cadherin 3, type 1, P-cadherin (placental) \\
\hline $4.00 \mathrm{E}-06$ & 11.028542 & FANCI & $\operatorname{chr} 15$ & Fanconi anemia, complementation group I \\
\hline 6.02E-05 & 10.597402 & SMC2 & chr9 & Structural maintenance of chromosomes 2 \\
\hline 0.00508388 & 10.348655 & KLHL6 & chr3 & Kelch-like 6 (Drosophila) \\
\hline $1.87 \mathrm{E}-06$ & 10.16844 & CENPW & chr6 & Centromere protein $\mathrm{W}$ \\
\hline 1.19E-04 & 10.124681 & KIF23 & chr15 & Kinesin family member 23 \\
\hline 0.00435048 & 10.109373 & CD52 & chr1 & CD52 molecule \\
\hline $1.90 \mathrm{E}-04$ & 10.10572 & C1QTNF6 & chr22 & C1q and tumor necrosis factor related protein 6 (C1QTNF6) \\
\hline
\end{tabular}

to synthesize cDNA using Reverse Transcription Reagents (Applied Byosystems, Foster City, CA, USA). qPCR for the selected genes was carried out as previously described. ${ }^{16}$ All qPCR reactions were performed in triplicate with MyiQ2 two-color real-time PCR detection system (Bio-Rad, Hercules, CA, USA), using SYBR Green (Applied Biosystems) as fluorescent dye, and normalized to $\beta$-actin mRNA levels. Data are presented as the mean \pm standard deviation. Primers for MMP-11 were designed using Primer3 software, while those specific for MMP-9, S100A2, and Clusterin were previously described. ${ }^{17-19}$ The sequences for the primers are the following: S100A2: 5'-CTGGCTGTGCTGGTCACTAC-3' (forward); 5'-TGGGCAGCTCCTTGTGCAGA-3' (reverse); MMP-9: 5'-GCACGACGTCTTCCAGTACC-3' (forward), 5'-CAG GATGTCATAGGTCACGTAGC-3' (reverse); MMP-11: 5'-CTCGTGGGTCCTGACTTCTT-3' (forward), 5'-GCAG TTGTCATGGTGGTTGT-3' (reverse); Clusterin: 5'-GAG CAGCTGAACGAGCAGTTT-3' (forward), 5'-CTTCGCC TTGCGTGAGGT-3' (reverse); $\beta$-Actin: 5'-CCCAGCACA ATGAAGATCAA-3' (forward), 5'-CGATCCACACGGA GTACTTG-3' (reverse).

\section{Immunohistochemistry}

Immunohistochemical staining was performed on 4- $\mu$ m-thick sections using standard techniques. Briefly, the slides were deparaffinized and antigen retrieval was performed by incubating the slides in hot sodium citrate, $\mathrm{pH}$ 6.0, for $30 \mathrm{~min}$. Slides were incubated for $10 \mathrm{~min}$ in $3 \%$ hydrogen peroxide, washed in distilled $\mathrm{H}_{2} \mathrm{O}$, and incubated for 30 min with $0.4 \%$ Triton X100/TBS pH 7.2. Non-specific binding was blocked by treating the slides with $4 \%$ normal goat serum in $0.1 \%$ Triton X100/TBS for $1 \mathrm{~h}$. Slides were then incubated overnight in the following rabbit anti-human primary antibodies: MMP-9 (Cell Signaling Technology, \#13667, Danvers, MA, USA), MMP-11 (Abcam, \#ab52904, Cambridge, MA, USA), S100A2 (Abcam, \#ab109494), and mouse anti-human Clusterin antibody (Pharmingen, clone E5, \#552886, San Jose, CA, USA). Secondary antibodies were purchased from Vector Laboratories, Burlingame, CA, USA (anti-rabbit: \#PK-6101; anti-mouse: \#PK6102) and diluted 1:200 in 2\% normal goat serum/0.1\% Triton X100/TBS. Formalin-fixed paraffin-embedded MCF7 and PANC1 cell pellets were used as positive control for MMP-11, while normal conjunctiva specimens were used as positive control for Clusterin staining. Incubations with no primary antibody as well as assessment of non-reactive stromal cells was used as negative control. Diaminobenzidine was used as chromogen and slides were counterstained with hematoxylin before mounting. Immunoreactivity was scored as follows: 0, no expression; 1+, weak expression; 2+, moderate expression; and $3+$, strong expression in over $50 \%$ of cells. 
Table 3 Genes downregulated in ocular surface squamous neoplasia compared with normal conjunctiva; fold change (FC) $>11$

\begin{tabular}{|c|c|c|c|c|}
\hline $\mathrm{P}$-value & $F C$ & Gene symbol & Chromosome & Gene name \\
\hline 2.00E-06 & 178.1298 & CLU & chr8 & Clusterin \\
\hline 1.87E-04 & 72.28282 & DKFZP547L112 & chr15 & Uncharacterized protein DKFZp547L112 \\
\hline 7.29E-06 & 45.74096 & KGFLP1 & chr9 & Fibroblast growth factor 7 pseudogene \\
\hline 4.59E-07 & 35.09851 & AQP5 & chr12 & Aquaporin 5 \\
\hline 0.001342 & 29.82204 & TCAP & chr17 & Titin-cap (telethonin) \\
\hline 4.44E-06 & 29.38312 & CPAMD8 & chr19 & C3 and PZP-like, $\alpha$-2-macroglobulin domain containing 8 \\
\hline 2.70E-04 & 25.80205 & CAPN5 & chr11 & Calpain 5 \\
\hline $2.25 \mathrm{E}-04$ & 25.23275 & MOP-1 & chr4 & MOP-1 \\
\hline 0.004186 & 24.81032 & HOPX & chr4 & HOP homeobox \\
\hline $1.12 \mathrm{E}-04$ & 22.25546 & LOC100507547 & chr6 & Uncharacterized LOC100507547 \\
\hline 0.002928 & 22.09582 & GDF15 & chr19 & Growth differentiation factor 15 \\
\hline 9.52E-05 & 21.93042 & SPARCL1 & chr4 & SPARC-like 1 (hevin) \\
\hline $5.25 \mathrm{E}-04$ & 20.52862 & SHOX & chrY & Short stature homeobox \\
\hline $1.80 \mathrm{E}-06$ & 18.92113 & PRB3 & chr12 & Proline-rich protein BstNI subfamily 3 \\
\hline 0.001213 & 18.75985 & ALDH3A1 & $\operatorname{chr} 17$ & Aldehyde dehydrogenase 3 family, member A1 \\
\hline $5.73 \mathrm{E}-04$ & 18.56191 & EFEMP1 & chr2 & EGF-containing fibulin-like extracellular matrix protein 1 \\
\hline 1.27E-04 & 17.88825 & ZNF497 & chr19 & Zinc finger protein 497 \\
\hline 4.19E-04 & 16.98899 & SCARA3 & chr8 & Scavenger receptor class $\mathrm{A}$, member 3 \\
\hline $3.95 \mathrm{E}-04$ & 14.59297 & CES1 & chr16 & Carboxylesterase 1 \\
\hline $3.31 \mathrm{E}-04$ & 14.19237 & CFHR3 & chr1 & Complement factor H-related 3 \\
\hline 4.47E-04 & 13.14154 & COL11A2 & chr6 & Collagen, type XI, alpha 2 \\
\hline $9.27 \mathrm{E}-04$ & 12.98495 & SOD3 & chr4 & Superoxide dismutase 3 \\
\hline $1.48 \mathrm{E}-04$ & 12.66532 & NR2F2 & chr15 & Nuclear receptor subfamily 2, group F, member 2 \\
\hline $8.24 \mathrm{E}-06$ & 12.66295 & CYBRD1 & chr2 & Cytochrome b reductase 1 \\
\hline $2.84 \mathrm{E}-05$ & 12.44124 & FLJ43860 & chr8 & FLJ43860 protein \\
\hline $3.54 \mathrm{E}-04$ & 12.43122 & KLF9 & chr9 & Kruppel-like factor 9 \\
\hline $5.69 \mathrm{E}-05$ & 12.3595 & ADD3 & chr10 & Adducin 3 (gamma) \\
\hline $7.90 \mathrm{E}-07$ & 12.33297 & LOC389834 & chr21 & Ankyrin repeat domain 57 pseudogene \\
\hline $1.08 \mathrm{E}-04$ & 12.3031 & COX7A1 & chr19 & Cytochrome c oxidase subunit VIIa polypeptide 1 (muscle) \\
\hline 0.001247 & 11.99697 & C11orf96 & chr11 & Chromosome 11 open reading frame 96 (C11orf96) \\
\hline 0.005758 & 11.75529 & HAPLN2 & chr1 & Hyaluronan and proteoglycan link protein 2 (HAPLN2) \\
\hline 4.05E-04 & 11.51033 & S1PR1 & chr1 & Sphingosine-1-phosphate receptor 1 (S1PR1) \\
\hline 4.70E-04 & 11.43265 & $\mathrm{CFH}$ & chr1 & Complement factor $\mathrm{H}(\mathrm{CFH})$ \\
\hline 0.003684 & 11.3695 & FAM48A & chr13 & Family with sequence similarity 48 , member A (FAM48A) \\
\hline 1.01E-04 & 11.21465 & PFDN6 & chr6 & Prefoldin subunit 6 \\
\hline 2.59E-05 & 11.21126 & NPB & chr17 & Neuropeptide B (NPB) \\
\hline
\end{tabular}

\section{Results}

\section{Clinical Characteristics}

Cases from our prior study ${ }^{15}$ with sufficient frozen tissue available for RNA extraction and gene expression profiling were used. These included one primary conjunctival carcinoma in situ, six primary, and two recurrent conjunctival squamous cell carcinomas resected at King Khaled Eye Specialist Hospital between 2005 and 2010. In some of the recurrent cases, cryotherapy had been used in addition to surgery, but no prior chemotherapy exposure was documented. Four of the patients were between 45 and 65 years of age, with the remainder over 65 years old; all patients, except one, were males. None of the patients were known to be immunosuppressed or have a history of HPV infection. Clinical features are described in Table 1 using the same case numbers as our prior publication ${ }^{15}$ to facilitate comparisons with those previously reported DNA alterations.

\section{Gene Expression Profile Data}

Microarray analysis was used to identify expression changes in the conjunctival squamous cell carcinoma and conjunctival carcinoma in situ cases as compared with normal conjunctiva. A large number of transcripts were upregulated in the tumors, and those with an increase of 10 -fold or more are listed in Table 2. Among the most upregulated genes associated with other types of epithelial malignancy, we observed induction of the matrix metalloproteinases, $M M P-9$ and $M M P-11$, which were increased by 38- and 33-fold, respectively, in the tumor samples as compared with normal controls. We also found overexpression of additional genes known to be elevated in other types of squamous cell carcinoma, such as collagen type I $\alpha 1$ (COL1A1), centromere protein F (CENPF), hexokinase 2 (HK2), and STAT1, which were increased by 33-, 25-, 22-, and 12-fold in the tumor cohort, respectively. A 22-fold induction of S100A2 was also noted. This calcium-binding protein important for the regulation of intracellular calcium signaling is known to be expressed in both mucosal carcinomas and in normal conjunctiva. ${ }^{20}$

Numerous reductions in expression were also identified in the carcinomas, and the 36 transcripts with a 11-fold or greater decrease as compared with normal conjunctiva are listed in Table 3 . The most downregulated gene was clusterin $(C L U)$, whose 
Table 4 Top canonical pathways and upstream regulators modulated by the genes overexpressed or downregulated in conjunctival squamous cell carcinoma; $P$-values were calculated using Fisher's Exact test

\begin{tabular}{lc} 
Name & P-value \\
\hline Top upregulated pathways & \\
RAN signaling & $4.56 \mathrm{E}-04$ \\
Hereditary breast cancer signaling & $5.04 \mathrm{E}-03$ \\
Cell cycle control of chromosomal replication & $5.47 \mathrm{E}-03$ \\
lnosine-5'-phosphate biosynthesis II & $6.41 \mathrm{E}-03$ \\
Purine nucleotides de novo biosynthesis II & $9.67 \mathrm{E}-03$ \\
& \\
& \\
Top downregulated pathways & $1.09 \mathrm{E}-03$ \\
Epithelial adherens junction signaling & $1.93 \mathrm{E}-02$ \\
Ceramide signaling & $2.00 \mathrm{E}-02$ \\
Cardiac-adrenergic signaling & $3.19 \mathrm{E}-02$ \\
Production of nitric oxide and reactive oxygen & \\
species in macrophages & $3.28 \mathrm{E}-02$
\end{tabular}

expression was reduced 178 -fold in the tumor samples. In addition to these more highly suppressed transcripts, the gene XPA, related to the DNA damage repair syndrome Xeroderma Pigmentosum, was downregulated 3.6-fold in the tumor cohort. This is consistent with our prior genomic study, which identified DNA deletions in conjunctival squamous cell carcinoma tumor specimens at loci encoding genes altered in Xeroderma Pigmentosum. ${ }^{15}$

\section{Confirmation of Selected Upregulated and Downregulated Genes}

Microarray results for selected loci were validated by quantitative PCR (qPCR) and immunohistochemistry. As shown in Figure 1a, MMP-9 gene expression was induced between 20- and 800-fold in the tumor cohort as compared with normal conjunctiva and limbus when assessed using qPCR in mRNA from the original snap-frozen cohort. Upregulation of MMP-11 was also confirmed by qPCR, with increases of up to 120-fold in the tumors (Figure 1b). Interestingly, the two tumors, which had less pronounced increases in MMP-11, were histopathologically distinctive. Case \#1 was an in situ lesion, and the frozen specimen may have contained more admixed normal tissues than the invasive carcinomas. Case \#10 showed features of adnexal differentiation, and had the lowest numbers of chromosomal alterations in our prior array-based comparative genomic hybridization analysis. ${ }^{15}$ The final upregulated locus we sought to confirm using qPCR was S100A2, in which this second technique revealed mRNA levels from 5 to 50 times than in normal conjunctiva and limbus (Figure 1c).

The most prominently reduced mRNA in the tumors analyzed by microarray was encoded by clusterin. This change was also confirmed by qPCR, although it was not as prominent as in the initial expression profiles, with a decrease in $C L U$ mRNA expression of 4- to 40 -fold as compared with normal conjunctival and limbal samples (Figure 1d).
Interestingly, the conjunctival carcinoma in situ (Case \#1) again had an expression level closer to that of the normal tissue than the invasive tumors.

In order to visualize the expression of proteins within neoplastic cells, and in particular the relationship between intraepithelial or in situ lesions and normal conjunctiva, we used immunohistochemistry. Four cases of severely dysplastic conjunctival intraepithelial neoplasia or conjunctival carcinoma in situ resected at Johns Hopkins Hospital were examined. For S100A2, strong expression was noted in the basal layer of normal conjunctiva where proliferating progenitors are located, as well as diffusely in conjunctival intraepithelial neoplasia and conjunctival carcinoma in situ (-) cells (Figure 2a). MMP-11 protein expression was also strong in dysplastic cells, with weaker and patchy staining in adjacent non-neoplastic conjunctiva (Figure 2b). Finally, in normal conjunctiva, clusterin protein expression was moderate and diffuse, while in the conjunctival intraepithelial neoplasia and conjunctival carcinoma in situ specimens, no immunoreactivity was noted (Figure 2c). For all three of these antibodies, expression in neoplastic cells and differences with normal conjunctiva were the same in all four cases examined.

\section{Pathway Analysis}

We used Ingenuity Pathway Analysis (IPA) to determine the top five signaling pathways that are regulated by the genes that we found to be overexpressed in the conjunctival squamous cell carcinoma cases compared with normal conjunctiva tissues. As summarized in Table 4, these pathways include RAN signaling, hereditary breast cancer signaling, chromosomal replication, inosine-5'-phosphate biosynthesis, and purine nucleotides de novo biosynthesis. Significantly elevated upstream regulators of these pathways included GLI1, TGF $\beta 1$, ERBB2, HSF1, and SPDEF, which might be potentially responsible for driving tumorigenesis in conjunctival squamous cell carcinoma. We also determined the top five signaling pathways modulated by the genes that we found to be downregulated in the conjunctival squamous cell carcinoma cases compared with normal conjunctiva tissues. These pathways include epithelial adherens junction signaling, ceramide signaling, cardiac-adrenergic signaling, nitric oxide production, and glutamine biosynthesis (Table 4). The upstream regulators of these pathways included SOX9, LAMTOR5, XBP1, NR1H3, and SOX4.

\section{Discussion}

Conjunctival squamous neoplasms are non-pigmented malignant tumors of the ocular surface. They are more frequent in countries located near the equator, with increased exposure to UV-B light, which is considered one of the main etiologic factors for the pathogenesis of the disease. ${ }^{3}$ Only limited information is known about 
a

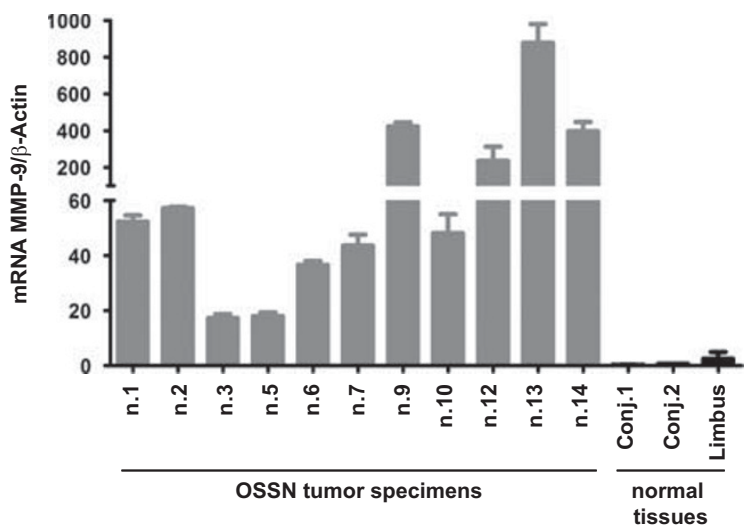

C

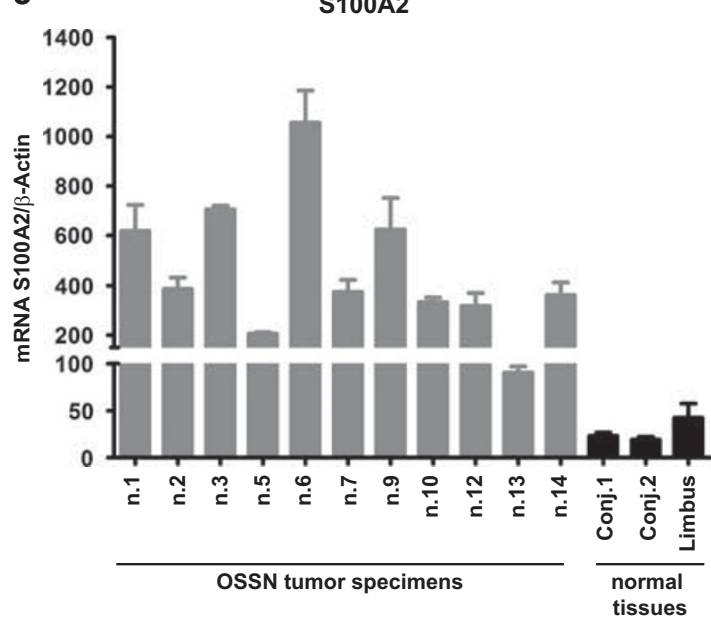

b

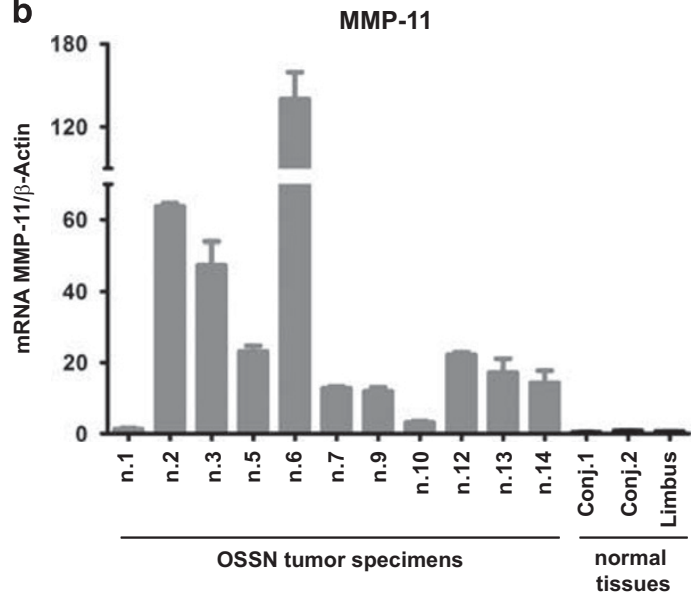

d

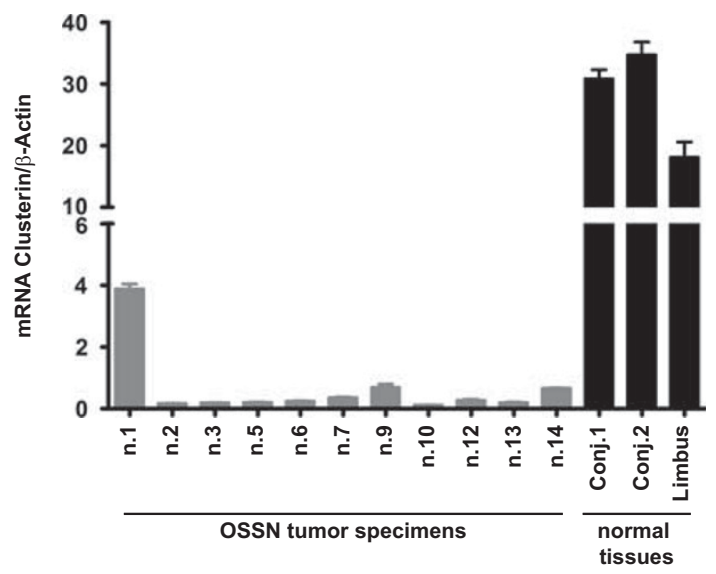

Figure 1 mRNA levels of MMP-9, MMP-11, S100A2, and Clusterin in ocular surface squamous neoplasia tumors vs normal conjunctiva and limbus. (a) mRNA levels of MMP-9 were induced more than 20-fold in the tumors as compared with normal conjunctiva and limbus, as determined by qPCR in 11 specimens of ocular surface squamous neoplasia. Cases \#6 and \#9 were not included in the microarray analysis and their clinical and pathological characteristics were previously described; ${ }^{15}$ (b) mRNA levels of MMP-11 were increased from 15- to 120-fold in the tumors as compared with normal conjunctiva and limbus, as determined by qPCR in 11 specimens of ocular surface squamous neoplasia; (c) mRNA levels of S100A2 were increased from 5- to 50-fold in tumor samples as compared with normal conjunctiva and limbus, as determined by qPCR in 11 specimens; (d) mRNA levels of clusterin were reduced from 4- to 40-fold in ocular surface squamous neoplasia tumors as compared with normal conjunctiva and limbus, as determined by qPCR in 11 specimens.

the molecular alterations that drive tumorigenesis. The main goal of our study was to characterize the transcriptional profile of conjunctival squamous cell carcinoma and compare it with normal conjunctiva in order to identify new diagnostic markers and therapeutic targets. We identified a number of dysregulated genes, many of which have been previously implicated in the pathobiology of other types of cancer.

Among the most upregulated genes were the matrix metalloproteinases, MMP-9 and MMP-11, whose mean expression was increased in tumors by 38- and 33-fold, respectively, compared with normal conjunctiva. Reports by $\mathrm{Ng}$ et $a \mathrm{l}^{21}$ and $\mathrm{Di}$ Girolamo et al. ${ }^{22}$ implicating MMPs in the pathogenesis of conjunctival squamous cell carcinoma have specifically investigated MMPs and their inhibitors by immunological methods and are in support of our findings. On the basis of their analysis of MMPs, which included MMP-9 and -11, Di Girolamo et al. proposed an imbalanced enzyme/inhibitor ratio which could promote invasive activity. MMPs belong to a family of closely related Zinc-finger endopeptidases, involved in the degradation of specific components of the extracellular matrix and basement membrane, promoting tumor invasion and metastasis. ${ }^{23}$ Their crucial role in the metastatic progression of the primary tumor has led to the discovery of MMP inhibitors for use as anticancer treatments.

The induction of MMP expression in other types of squamous cell carcinoma has been reported in previous studies, including cutaneous squamous cell carcinoma, where MMP-2 and MMP-9 expression was found to correlate with depth of lesion, inflammation, and microvessel density. ${ }^{24}$ In another 


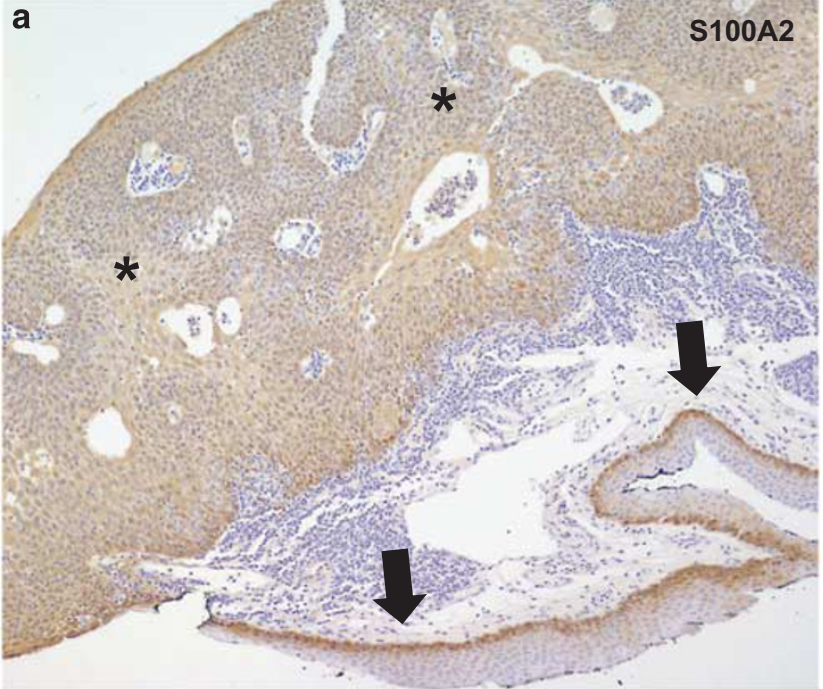

b
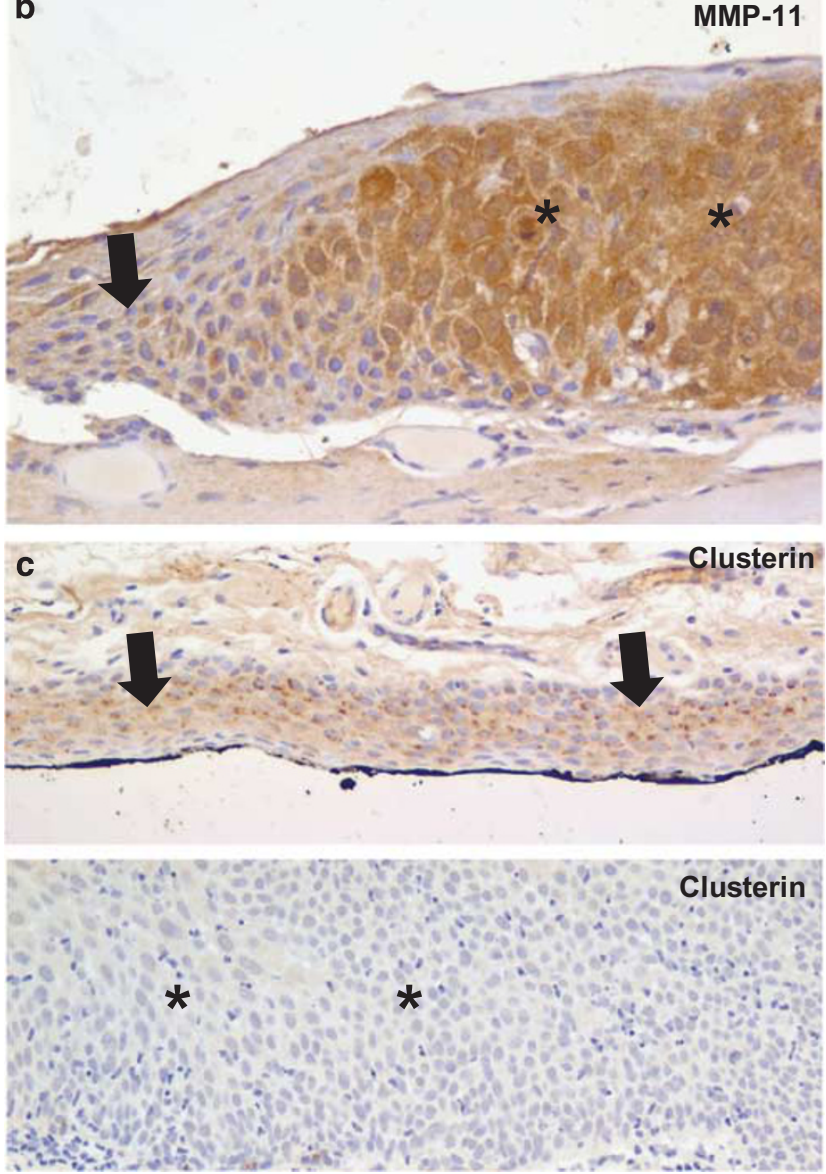

Figure 2 Immunohistochemical analysis of conjunctival intraepithelial neoplasia and carcinoma in situ. (a) S100A2 is expressed in the epithelial base of normal conjunctiva present in the bottom of the panel (arrows), and more diffusely in the conjunctival carcinoma in situ (asterisks; original magnification $100 \times$ ). (b) The dysplastic portion of the conjunctival epithelium (asterisks) is strongly positive for MMP-11, while weaker immunoreactivity is noted in the less atypical cells adjacent to the lesion (arrow; original magnification $400 \times$ ). (c) Clusterin shows diffuse moderate expression in the normal conjunctival epithelium in the upper panel (arrows), while no expression was present in an adjacent conjunctival carcinoma in situ shown in the lower panel (asterisks; original magnification $200 \times$ ). report, high expression of MMP-9 was observed in the tumor stroma of squamous cell carcinoma of the skin, providing evidence for the involvement of MMP-9 in tumor invasiveness of keratinocytederived tumors. ${ }^{25}$ Interestingly, in head and neck squamous cell carcinomas, MMP-9 immunoreactivity has been observed in $82 \%$ of the cases, and is associated with shortened relapse-free survival, with high MMP-9 expression linked to more aggressive relapses. ${ }^{26}$ Stromelysin-3 (ST3/MMP-11) is also associated with human tumor progression. Elevated expression of MMP-11 has been observed in carcinomas with poor prognosis, whereas in benign tumors, MMP-11 is mildly detectable or not expressed at all. ${ }^{27}$ Interestingly, MMP-11 protein has been demonstrated using immunohistochemistry in $70 \%$ of oral squamous cell carcinoma, where it is associated with lymph node involvement, but it is not a significant prognostic indicator. ${ }^{28}$

Additional upregulated loci we identified previously associated with related cancers include collagen type I $\alpha 1$ (COL1A1), which is known to be induced in oral squamous cell carcinoma, where it has an important role in distinguishing oral squamous cell carcinoma from controls. $^{29}$ Our transcriptional profile analysis also showed upregulation of centromere protein $\mathrm{F}$ (CENPF) and hexokinase 2 (HK2), which are known to be overexpressed in esophageal ${ }^{30}$ and cervical squamous cell carcinoma. Finally, the expression of HK2 is associated with radiation resistance. ${ }^{31}$

An additional gene meriting further investigation is the calcium-binding protein S100A2, which was previously shown to be expressed in the normal epithelium of the ocular surface. ${ }^{20}$ We found that this gene was upregulated more than 20-fold in our tumor cohort compared with normal conjunctiva, and confirmed expression of the S100A2 protein in both the basal proliferating layer of non-neoplastic epithelium and more diffusely in tumor cells. Our data are consistent with the previous report which used immunofluorescence to show high S100A2 expression in ocular surface carcinomas. ${ }^{20}$ These investigators also documented a reduction in S100A2 levels as limbal stem cells reduced their proliferative capacity and differentiated. ${ }^{20}$ Together with this prior study, our findings support the concept that S100A2 may have an important role in promoting proliferation and inhibiting differentiation of the normal conjunctiva and limbus, and that its overexpression is a common feature of neoplastic transformation in these tissues.

The most downregulated gene in our conjunctival squamous cell carcinoma cohort was Clusterin $(C L U)$, which is also known as apolipoprotein $\mathrm{J}$. This multifunctional protein is associated with clearance of cellular debris and apoptosis, as well as with lipid transport, membrane recycling, cell adhesion, tumor progression, and neurodegenerative disorders. ${ }^{32}$ Clusterin is known to be expressed in the ocular surface epithelium, but it is significantly 
reduced in the keratinized conjunctiva, suggesting its potential role in maintaining the ocular surface epithelium in a non-keratinizing state. ${ }^{33,34}$ In addition, it has been found that Clusterin strongly binds MMP-9, inhibiting its enzymatic activity and MMP-9-mediated degradation of the tight junction structures formed between human epithelial cells, thereby preventing tumor invasion. ${ }^{35}$ However, as we had only two cases of recurrent conjunctival squamous cell carcinoma in our tumor cohort, we cannot infer at the moment any statistical association between the expression of the most upregulated or downregulated genes and the clinical outcome or progression of the disease.

By performing pathway analysis, we observed that the epithelial adherens junction signaling was the most downregulated signaling pathway in the tumor cohort. Interestingly, the expression of Notch1 receptor, one of the genes related with this pathway, was reduced 2.7 -fold in the conjunctival squamous cell carcinoma samples compared with normal conjunctiva tissues. It has been shown that Notch1 has an oncosuppressor role in the skin by inhibiting $\beta$-catenin signaling. ${ }^{36}$ Therefore, the downregulation of this receptor that we observed in the conjunctival squamous cell carcinoma cases suggests that Notch1 might have a tumor suppressor function also in the SCC of the ocular surface, and its upregulation might represent a new potential therapeutic strategy in the treatment of conjunctival squamous cell carcinoma.

In summary, here, we present evidence for dysregulation of several genes associated with epithelial tumorigenesis in the ocular surface by gene expression analysis. We provide further validation of the gene expression data by qPCR and immunohistochemistry for four genes, MMP-9, MMP-11, S100A2, and Clusterin. Overall, gene dysregulations observed in our samples show multiple hallmarks of cancer including proliferation and invasion. Further investigations would be needed to understand the significance of individual aberrations as well as the pathways that they disrupt. Thus far, such a global gene expression study has not been reported for ocular surface squamous neoplasia and together with our previous array-based comparative genomic hybridization study, we report a comprehensive analysis of chromosomal and transcriptional aberrations in the same set of tumors. Our data form the basis for future investigations to delineate the molecular mechanisms underlying the development and progression of ocular surface squamous neoplasia.

\section{Acknowledgments}

This study was supported by King Khaled Eye Specialist Hospital - Wilmer Eye Institute Collaborative Research Grant and by Research to Prevent Blindness Wilmer Eye Institute. Sample quality assessment and microarray analysis were conducted at The Sidney Kimmel Cancer Center Microarray Core Facility at the Johns Hopkins University, supported by NIH grant P30 CA006973 entitled Regional Oncology Research Center.

\section{Disclosure/conflict of interest}

The authors declare no conflict of interest.

\section{References}

1 Kiire CA, Srinivasan S, Karp CL. Ocular surface squamous neoplasia. Int Ophthalmol Clin 2010;50: 35-46.

2 Yang J, Foster CS. Squamous cell carcinoma of the conjunctiva. Int Ophthalmol Clin 1997;37:73-85.

3 Lee GA, Hirst LW. Ocular surface squamous neoplasia. Surv Ophthalmol 1995;39:429-450.

4 Shields CL, Ramasubramanian A, Mellen PL et al. Conjunctival squamous cell carcinoma arising in immunosuppressed patients (organ transplant, human immunodeficiency virus infection). Ophthalmology 2011;118: 2133-2137.

5 Ateenyi-Agaba C, Franceschi S, Wabwire-Mangen F et al. Human papillomavirus infection and squamous cell carcinoma of the conjunctiva. Br J Cancer 2010;102: 262-267.

6 Peralta R, Valdivia A, Estañol P et al. Low frequency of human papillomavirus infection in conjunctival squamous cell carcinoma of Mexican patients. Infect Agent Cancer 2011;6:24.

7 Tunc M, Char DH, Crawford B et al. Intraepithelial and invasive squamous cell carcinoma of the conjunctiva: analysis of 60 cases. Br J Ophthalmol 1999;83:98-103.

8 McKelvie PA, Daniell M, McNab A et al. Squamous cell carcinoma of the conjunctiva: a series of 26 cases. Br J Ophthalmol 2002;86:168-173.

9 Shields CL, Naseripour M, Shields JA. Topical mitomycin $\mathrm{C}$ for extensive, recurrent conjunctival-corneal squamous cell carcinoma. Am J Ophthalmol 2002;133: 601-606.

10 Midena E, Angeli CD, Valenti M et al. Treatment of conjunctival squamous cell carcinoma with topical 5fluorouracil. Br J Ophthalmol 2000;84:268-272.

11 Boehm MD, Huang AJ. Treatment of recurrent corneal and conjunctival intraepithelial neoplasia with topical interferon alfa 2b. Ophthalmology 2004;111:1755-1761.

12 Schechter BA, Koreishi AF, Karp CL et al. Long-term follow-up of conjunctival and corneal intraepithelial neoplasia treated with topical interferon alfa-2b. Ophthalmology 2008;115:1291-1296 1296.e1.

13 Smith ML, Ford JM, Hollander MC et al. p53-mediated DNA repair responses to UV radiation: studies of mouse cells lacking p53, p21, and/or gadd45 genes. Mol Cell Biol 2000;20:3705-3714.

14 Mahomed A, Chetty R. Human immunodeficiency virus infection, Bcl-2, p53 protein, and Ki-67 analysis in ocular surface squamous neoplasia. Arch Ophthalmol 2002;120:554-558.

15 Asnaghi L, Alkatan H, Mahale A et al. Identification of multiple DNA copy number alterations including frequent 8p11.22 amplification in conjunctival squamous cell carcinoma. Invest Ophthalmol Vis Sci 2014;55:8604-8613. 
16 Asnaghi L, Ebrahimi KB, Schreck KC et al. Notch signaling promotes growth and invasion in uveal melanoma. Clin Cancer Res 2012;18:654-665.

17 Matsubara D, Niki T, Ishikawa S et al. Differential expression of S100A2 and S100A4 in lung adenocarcinomas: clinicopathological significance, relationship to p53 and identification of their target genes. Cancer Sci 2005;96:844-857.

18 Safranek J, Pesta M, Holubec L et al. Expression of MMP-7, MMP-9, TIMP-1 and TIMP-2 mRNA in lung tissue of patients with non-small cell lung cancer (NSCLC) and benign pulmonary disease. Anticancer Res 2009;29:2513-2517.

19 Cochrane DR, Wang Z, Muramaki M et al. Differential regulation of clusterin and its isoforms by androgens in prostate cells. J Biol Chem 2007;282:2278-2287.

20 Li J, Riau AK, Setiawan M et al. S100A expression in normal corneal-limbal epithelial cells and ocular surface squamous cell carcinoma tissue. Mol Vis 2011;17: 2263-2271.

$21 \mathrm{Ng} \mathrm{J,} \mathrm{Coroneo} \mathrm{MT,} \mathrm{Wakefield} \mathrm{D} \mathrm{et} \mathrm{al.} \mathrm{Ultraviolet}$ radiation and the role of matrix metalloproteinases in the pathogenesis of ocular surface squamous neoplasia. Invest Ophthalmol Vis Sci 2008;49:5295-5306.

22 Di Girolamo N, Atik A, McCluskey PJ et al. Matrix metalloproteinases and their inhibitors in squamous cell carcinoma of the conjunctiva. Ocul Surf 2013;11: 193-205.

23 Curran S, Murray GI. Matrix metalloproteinases: molecular aspects of their roles in tumour invasion and metastasis. Eur J Cancer 2000;36:1621-1630.

24 O'Grady A, Dunne C, O'Kelly $\mathrm{P}$ et al. Differential expression of matrix metalloproteinase (MMP)-2, MMP-9 and tissue inhibitor of metalloproteinase (TIMP)-1 and TIMP-2 in non-melanoma skin cancer: implications for tumour progression. Histopathology 2007;51:793-804.

25 Poswar FO, Fraga CA, Farias LC et al. Immunohistochemical analysis of TIMP-3 and MMP-9 in actinic keratosis, squamous cell carcinoma of the skin, and basal cell carcinoma. Pathol Res Pract 2013;209:705-709.
26 Ruokolainen H, Pääkkö P, Turpeenniemi-Hujanen T. Expression of matrix metalloproteinase- 9 in head and neck squamous cell carcinoma: a potential marker for prognosis. Clin Cancer Res 2004;10:3110-3116.

27 Asch PH, Basset P, Roos $\mathrm{M}$ et al. Expression of stromelysin 3 in keratoarcanthoma and squamous cell carcinoma. Am J Dermatopathol 1999;21:146-150.

28 Soni S, Mathur M, Shukla NK et al. Stromelysin-3 expression is an early event in human oral tumorigenesis. Int J Cancer 2003;107:309-316.

29 Chen C, Méndez E, Houck J et al. Gene expression profiling identifies genes predictive of oral squamous cell carcinoma. Cancer Epidemiol Biomarkers Prev 2008;17:2152-2162.

30 Pimkhaokham A, Shimada Y, Fukuda Y et al. Nonrandom chromosomal imbalances in esophageal squamous cell carcinoma cell lines: possible involvement of the ATF3 and CENPF genes in the 1q32 amplicon. Jpn J Cancer Res 2000;91:1126-1133.

31 Huang $\mathrm{X}$, Liu $\mathrm{M}$, Sun $\mathrm{H}$ et al. HK2 is a radiation resistant and independent negative prognostic factor forpatients with locally advanced cervical squamous cell carcinoma. Int J Clin Exp Pathol 2015;8:4054-4063.

32 Koltai T. Clusterin: a key player in cancer chemoresistance and its inhibition. Onco Targets Ther 2014;7: 447-456.

33 Nishida K, Kawasaki S, Kinoshita S. Clusterin may be essential for maintaining ocular surface epithelium as a non-keratinizing epithelium. Adv Exp Med Biol 1998;438:629-635.

34 Nakamura T, Nishida K, Dota A et al. Changes in conjunctival clusterin expression in severe ocular surface disease. Invest Ophthalmol Vis Sci 2002;43:1702-1707.

35 Jeong S, Ledee DR, Gordon GM et al. Interaction of clusterin and matrix metalloproteinase-9 and its implication for epithelial homeostasis and inflammation. Am J Pathol 2012;180:2028-2039.

36 Nicolas M, Wolfer A, Raj K et al. Notch1 functions as a tumor suppressor in mouse skin. Nat Genet 2003;33: 416-421. 